\title{
A SENSITIVITY-BASED COMMONALITY STRATEGY FOR FAMILY PRODUCTS OF MILD VARIATION, WITH APPLICATION TO AUTOMOTIVE BODY STRUCTURES
}

\author{
Ryan Fellini, Michael Kokkolaras, Nestor Michelena \\ $\{$ rfellini,mk, nestorm, pyp, kazu\} @umich.edu \\ Department of Mechanical Engineering, University of Michigan \\ G.G. Brown Bldg., Ann Arbor, Michigan 48109-2125 \\ Alexis Perez-Duarte* \\ alexispz@umich.edu \\ Department of Aerospace Engineering, University of Michigan \\ F.-X. Bagnoud Bldg., Ann Arbor, Michigan 48109-2140 \\ Peter A. Fenyes ${ }^{\|}$ \\ peter.a.fenyes@gm.com \\ General Motors R\&D and Planning, Vehicle Analysis \& Dynamics Lab \\ 30500 Mound Road, Warren, Michigan 48090-9055
}

\begin{abstract}
Identification of the product platform is a key step in designing a family of products. This article presents a methodology for selecting the product platform using information obtained from the individual optimization of the product variants. Under the assumption that the product variety requires only mild design changes, a sharing penalty vector (SPV) is derived by taking into consideration individual optimal designs and sensitivities of functional requirements. Commonality decisions are based on SPV values and the product family is designed optimally with respect to the chosen platform. An automotive body structure problem is used to demonstrate the proposed methodology. Variants are defined by changing the functional requirements they need to satisfy and/or the geometry of the associated finite element models.
\end{abstract}

\section{INTRODUCTION}

Sharing components within a family of products can be an effective method for corporations to increase cost savings (Meyer and Lehnerd, 1997; Ericsson and Erixon, 1999). A drawback to com-

\footnotetext{
${ }^{*}$ Graduate Student

${ }^{\dagger}$ Research Fellow

$¥$ Associate Research Scientist

$\S$ Professor

I Assistant Professor

॥ Staff Research Engineer
}

monality is that a sharing penalty can be incurred with regard to optimized individual product design. The challenge is to choose which components to share (i.e., define the product platform), and design the product family with minimal individual variation from optimal design.

Simpson et al. (1999) proposed a method for product platform synthesis and exploration based on a market segmentation grid and leveraging and scaling concepts (Meyer and Lehnerd, 1997). They solved the family design problem by means of compromise decision support problems using goal programming. This methodology has been built upon in a number of subsequent publications (Messac et al., 2000; Conner et al., 1999; Nayak et al., 2000).

Gonzalez-Zugasti et al. (1998) presented a method that uses cost gain models as the driving force for designing the product platform while satisfying performance and budget constraints: $a$ priori specified platforms are optimized first; family variants are designed second. Gonzalez-Zugasti and Otto (2000) formulated a design optimization problem for modular product architecture that can be solved to simultaneously determine module designs and their combination for the variant instantiations. Fujita et al. (2001) proposed a method for simultaneous optimization of module attributes and combinations. The modular architecture of the product family is fixed in both of the latter papers. 
Siddique et al. (1998) examined the applicability of product variety concepts to automotive design. In particular, they investigated whether product variety design concepts such as standardization, delayed differentiation, modularity, module interfaces, robustness, and mutability can be utilized. Keeping in mind that they limited their consideration for platform only to the underbody structure of a vehicle, they came to the conclusion that some of these concepts cannot be applied, mainly because of the integral nature of its architecture. However, they did mention the possibility of partitioning the underbody platform into major manufacturable modules that can be assembled.

Nelson et al. (2001) formulated platform design as a multiobjective optimal design problem by means of Pareto set theory. Given a fixed platform, a set of optimal points is generated based on the importance of the conflicting variant objectives. The designer can identify trade-offs, evaluate multiple platforms, and then make related decisions. Fellini et al. (2000) applied this concept to the design of an automotive product family based on a powertrain platform along with examining the hierarchical structure of the platform design problem. Kokkolaras et al. (2002) extended the target cascading formulation to the design of product families with pre-specified platforms. Both common and individual components, subsystems, and/or systems of the family products were designed optimally with respect to family and variant targets.

In the present work a methodology is proposed for making commonality decisions based on individual optima and sensitivity analysis of functional requirements. Emphasis is put on families of vehicle body structures using modelling approaches proposed by Fenyes (2000). We assume that we are dealing only with "mild variants", so that design changes can be guided by sensitivity information reasonably well.

The paper is organized as follows: Platformbased design of body structures is introduced in the next section. The mathematical derivation underlying the proposed approach is presented. The methodology based on this derivation is formulated and demonstrated by means of an automobile body structure case study. Results are discussed and conclusions are drawn.

\section{Platform-Based Design of Body STRUCTURES}

A component is defined as a manufactured object that is the smallest (indivisible) element of an assembly, and is described by a set of design vari- ables. A product is an artifact made up of components. A product platform is the set of all components, manufacturing processes, and/or assembly steps that are common in a set of products. A product family is the set of products that are built upon a product platform. A product in a product family is also referred to as a product variant. Two types of sharing are possible when selecting a product platform that is not based on manufacturing processes or assembly steps. In component sharing, one or more components are common across a family of products as shown in Figure 1. In addition, it is possible to share "scaled"

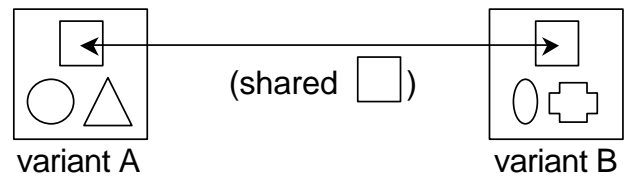

Figure 1: Platform-based products (component sharing).

versions of components. Mathematically this can be described as design variable sharing, where the components themselves are derived from a platform. The example in Figure 2 shows the crosssection of two structural beam elements. While

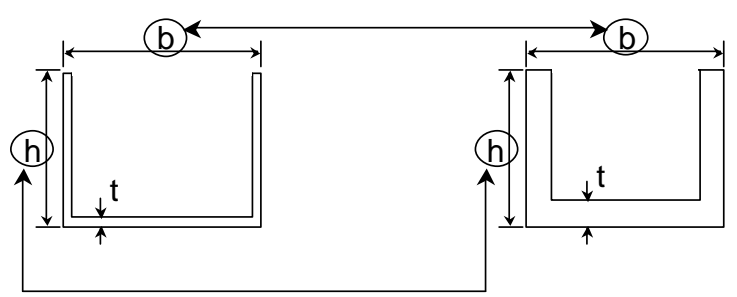

(shared b \& h)

Figure 2: Platform-based components (design variable sharing).

the height and width of both parts are the same, the thickness is different. The product containing the "thicker" component variant has higher rigidity requirements. By not sharing the thickness, the other product with lesser rigidity requirements does not have to take on an unnecessarily large weight penalty. The manufacturing advantage can also be illustrated by this example. By keeping width and height invariant, the same stamping equipment can be used with different gauge steel. In general, manufacturing considerations should be taken into account in the design of platforms. We do not address this aspect explicitly but we attempt to recognize the associated design impact. 


\subsection{Problem Formulation}

The following definitions are necessary to formulate the variant and family design problems:

$\mathcal{P}=\{A, B, C, \ldots\}:$ set of $m$ products

$\boldsymbol{x}^{p}:$ vector of design variables for product $p \in \mathcal{P}$

$\mathcal{S}$ : set of indices describing a platform

$\mathbb{S}$ : set of all permissible platforms

$\mathcal{S}^{*}$ : set of indices describing the optimal platform

$\boldsymbol{x}^{p, \circ}$ : null platform optimal design of product $p$, corresponding to the individual optimal designs solutions of Problem (1) below

$\boldsymbol{x}^{p, *}$ : family optimal design of product $p$, solution of Problem (2) below. Because of the sharing of variables whose indices are in $\mathcal{S}$, for $p, q \in \mathcal{P}$ and $i \in \mathcal{S}$, we have $x_{i}^{p, *} \equiv x_{i}^{q, *}=x_{i}^{*}$

$\mathcal{C}^{p}$ : set of indices of the active constraints at the null platform optimum of product $p$

For convenience, equality constraints are assumed to have been eliminated implicitly or explicitly. The individual optimal design problem for product variant $p$ can be formulated as follows:

$$
\begin{array}{rc}
\min _{\boldsymbol{x}^{p}} & f^{p}\left(\boldsymbol{x}^{p}\right) \\
\text { subject to } & \mathbf{g}^{p}\left(\boldsymbol{x}^{p}\right) \leq \mathbf{0}
\end{array}
$$

The family design problem is then formulated accordingly as

$$
\begin{array}{cc}
\min _{\boldsymbol{x}^{A}, \boldsymbol{x}^{B}, \ldots} & \left\{f^{p}\left(\boldsymbol{x}^{p}\right) \quad \mid \quad p \in \mathcal{P}\right\} \\
\text { subject to } & \mathbf{g}^{p}\left(\boldsymbol{x}^{p}\right) \leq \mathbf{0} \quad p \in \mathcal{P} \\
& x_{i}^{p}=x_{i}^{q} \quad i \in \mathcal{S}, \quad p, q \in \mathcal{P}, \quad p<q
\end{array}
$$

The platform selection methodology can be summarized as follows: Quantify sharing penalty by considering individual optimal designs and sensitivities of functional requirements; decide which components can be shared (i.e., determine the platform) with minimal sharing penalty; optimally design the product family around the chosen platform.

\section{Commonality Decisions}

The proposed approach is based on the use of optimality and sensitivity information obtained from individual product optimization to assess the potential sharing penalty incurred by sharing variables. When the products in the family contain a large number of components that are candidates for sharing, platform selection entails the solution of a large combinatorial problem. In the approach proposed, this problem is reduced to a simpler one under the assumptions listed below. The derivation presented in the following section is based on first order Taylor series approximation. Therefore, in order for the approximation to remain reasonably accurate, the general condition is that the individual optimal designs lie not "too far away" from each other so that the linear approximation is valid in the region between them. The derivation will be presented for a family of two products $A$ and $B$.

\subsection{Assumptions}

1. Self-sharing (i.e., component sharing within the same variant) is not possible. Furthermore, sharing is done on a same component basis.

2. Components are either shared by all family products or not at all.

3. Null platform optimal designs lie "close enough" to each other .

4. The platform design (denoted by superscript ${ }^{*}$ ) lies in the convex hull of the individual solutions (denoted by superscripts $p, \circ$ and $\left.{ }^{q, \circ}\right)$. It implies there exists $\alpha \in\left[\begin{array}{ll}0 & 1\end{array}\right]$ such that: $\forall i \in \mathcal{S}, \quad x_{i}^{*}=\alpha x_{i}^{p, \circ}+(1-\alpha) x_{i}^{q, \circ}$.

5. Constraint activity remains unchanged between individual and family design problems.

We refer to the design solutions that satisfy these assumptions as "mild variants".

\subsection{Sharing Penalty Vector Derivation}

Sharing may cause a penalty with respect to the individually optimized products, which is measured by the responses representing the functional requirements. In the context of the approach introduced in this article, the sharing decision consists essentially in deciding on the number $n$ of variables to share. The actual selection of an optimal platform can then be done by minimizing the relative variation of the designs based on any platform with $n$ shared variables with respect to the null platform optimal designs — while remaining in the feasible space for the variants. Formally, this translates to:

$$
\min _{\substack{\mathcal{S} \in \mathbb{S} \\ \text { subject to }}}|\mathcal{S}|=n
$$


where $\Delta=\Delta^{A}+\Delta^{B}$, and for $p \in \mathcal{P}$ :

$$
\Delta^{p}=\left|f^{p}\left(\boldsymbol{x}^{p}\right)-f^{p}\left(\boldsymbol{x}^{p, \circ}\right)\right|+\sum_{j \in \mathcal{C}^{p}} \max \left(g_{j}^{p}\left(\boldsymbol{x}^{p}\right), 0\right)
$$

In order to enable the meaningful summation of responses of different natures, we consider them to be non-dimensional.

A first order Taylor approximation of the variation in each response $f^{p}, g^{p}$ is introduced in agreement with the assumptions described in Section 3.1:

$$
\begin{aligned}
f^{p}\left(\boldsymbol{x}^{p}\right)-f^{p}\left(\boldsymbol{x}^{p, \circ}\right) & \approx \nabla f^{p, \circ}\left(\boldsymbol{x}^{p}-\boldsymbol{x}^{p, \circ}\right) \\
\text { for } j \in \mathcal{C}^{p}, g_{j}^{p}\left(\boldsymbol{x}^{p}\right) & \approx \nabla g_{j}{ }^{p, \circ}\left(\boldsymbol{x}^{p}-\boldsymbol{x}^{p, \circ}\right),
\end{aligned}
$$

where $\nabla f^{p, o}$ is the gradient of $f$ evaluated for the null platform optimal design of product $p$. By definition of $\mathcal{C}^{p}, g_{j}^{p}$ is active at the null platform optimum $\boldsymbol{x}^{p, \circ}$ therefore $g_{j}^{p}\left(\boldsymbol{x}^{p, \circ}\right)=0$.

Furthermore, under Assumption 4, the relation between the shared variables and the null platform can be rewritten as:

For $i \in \mathcal{S}, \quad\left(x_{i}^{*}-x_{i}^{A, \circ}\right)=(1-\alpha)\left(x_{i}^{B, \circ}-x_{i}^{A, \circ}\right)$

Consequently, the variation (sharing penalty) of the objective $f$ in one variant $A$ due to the sharing of the components in $\mathcal{S}$ is approximated by:

$$
\begin{aligned}
f^{A}\left(\boldsymbol{x}^{*}\right) & -f^{A}\left(\boldsymbol{x}^{A, \circ}\right) \\
& \approx \sum_{i \in \mathcal{S}} \nabla_{i} f^{A, \circ}\left(x_{i}^{*}-x_{i}^{A, \circ}\right) \\
& \approx(1-\alpha) \sum_{i \in \mathcal{S}} \nabla_{i} f^{A, \circ}\left(x_{i}^{B, \circ}-x_{i}^{A, \circ}\right)
\end{aligned}
$$

Letting $\delta_{i}=\left|x_{i}^{B, \circ}-x_{i}^{A, \circ}\right|$, an upper bound on the total variation in responses $\Delta^{A}$ is:

$$
\begin{aligned}
\Delta^{A} \leq & (1-\alpha) \sum_{i \in S}\left(\left|\nabla_{i} f^{A, \circ}\right| \delta_{i}\right. \\
& \left.+\sum_{j \in \mathcal{C}^{A}} \max \left(\nabla_{i} g_{j}{ }^{A, \circ} \delta_{i}, 0\right)\right)
\end{aligned}
$$

A similar upper bound can be obtained for $\Delta^{B}$. The Sharing Penalty Vector is then defined as the vector $\mathrm{SPV}$ with components $\mathrm{SPV}_{i}$ given as follows:

$$
\begin{aligned}
& \mathrm{SPV}_{i}= \\
& (1-\alpha)\left(\left|\nabla f_{i}^{A, \circ}\right| \delta_{i}+\sum_{j \in \mathcal{C}^{A}} \max \left(\nabla g_{j}{ }^{A, \circ} \delta_{i}, 0\right)\right) \\
& +\alpha\left(\left|\nabla f_{i}^{B, \circ}\right| \delta_{i}+\sum_{j \in \mathcal{C}^{B}} \max \left(\nabla g_{j}{ }^{B, \circ} \delta_{i}, 0\right)\right)
\end{aligned}
$$

\subsection{Application to Platform Decision}

The SPV provides an upper bound on the sharing penalty $\Delta$ through the aggregate sum of the $\mathrm{SPV}_{i}$ which correspond to a shared variable:

$$
\Delta \leq \sum_{i \in \mathcal{S}} \mathrm{SPV}_{i}
$$

The approach adopted in this article for approximating a solution to the original problem described in Eq. (3) is to minimize the upper bound on $\Delta$ given by the SPV as in Eq. (6). In this regard, the choice of the parameter $\alpha$ has to be discussed.

This parameter is theoretically determined by the position of the family solution for a given platform $\mathcal{S}$ relative to the position of the null platform solutions for the two variants ( $c f$. Assumption 4). In the framework described here, the exact value of $\alpha$ is not known since the solution to the family problem is not available. This issue can be bypassed through several different approximations. Taking the average of the SPV with $\alpha$ varying in $[0,1]$ yields $\alpha=1 / 2 ; \alpha$ can also be chosen so as to balance the highest sensitivity of $f^{A}$ and $f^{B}$; or different values can be tested and the resulting SPVs compared.

The design variables can then be arranged in order of increasing $\mathrm{SPV}_{i}$. Thus the design of the platform consists only in the decision of the number $n$ of variables that are to be shared. The actual shared variables are given by the first $n$ variables ordered according to the $\mathrm{SPV}_{i}$, and this minimizes the upper bound on $\Delta$.

\section{Proposed Methodology}

The proposed general methodology for selecting the product platform and designing the product family is as follows:

1. Generate product variants based on:

a. design requirements,

b. geometry of the model(s) ${ }^{1}$,

c. or both.

2. Develop appropriate analysis models and identify inputs and outputs.

3. Formulate and solve the optimal design problem for each variant, i.e., find null platform optimal designs, $c f$. Eq. (1).

4. Compare optimal design variable and sensitivity information by selecting $\alpha$ and computing the sharing penalty vector (SPV) using Eq. (5).

\footnotetext{
${ }^{1}$ i.e., no topological changes
} 
5. Arrange the variables in order of increasing $\mathrm{SPV}_{i}$.

6. Using the SPV, decide which components to share or not share.

7. Formulate and solve the family design problem with the chosen platform ( $c f$. Eq. (2)).

8. Compare family optimal designs to individual variant optimal designs and evaluate sharing penalty (iterate if necessary).

\section{Application Study}

A family of automotive body structures is considered. A variant is defined as a structure associated with specific dimensional properties (lengths) and functional requirements.

\subsection{Model Description}

The structures are modelled using finite elements in MSC-NASTRAN according to the MDO system described in Fenyes (2000) (cf. Figure 3). Modal and static loadcases (torsion on the front and rear shock towers, and bending) are considered, as shown in Figure 3. It is assumed that these load cases give access to the properties that the designer wishes to tailor, and therefore are valid as a basis of the design.

The finite element analysis outputs mass $(\mathrm{m})$ and natural frequencies $(\omega)$, in addition to displacements and stress responses for static load cases of front torsion, rear torsion and bending (denoted $d_{f t}, d_{r t}, d_{b}$ ) along with corresponding sensitivity information for all the design variables. These are the cross-sectional dimensions of the beams (width $b$, height $h$, and thickness $t$ ) and thicknesses $t$ of the shells. There are 66 design variables.

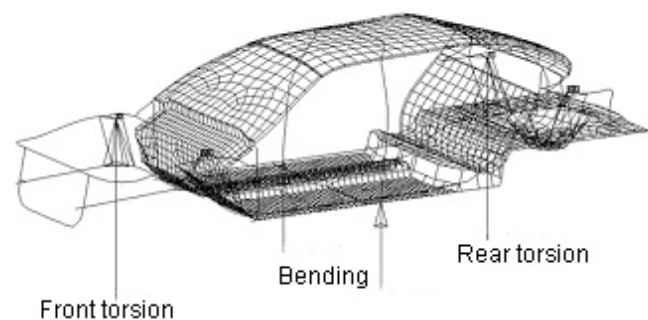

Figure 3: Automotive body structure model.

As mentioned, variants are generated either by implementing dimensional changes or by imposing different design requirements. We examine these two cases next.
Table 1: Null platform optima.

\begin{tabular}{|c|c|c|}
\cline { 2 - 3 } \multicolumn{1}{c|}{} & short & long \\
\hline mass $(\mathrm{kg})$ & 715.13 & 703.36 \\
\hline$\omega_{1}(\mathrm{~Hz})$ & 21.00 & 22.06 \\
\hline$\omega_{2}(\mathrm{~Hz})$ & 24.82 & 27.00 \\
\hline$d_{f t}(\mathrm{~mm})$ & 2.158 & 2.1698 \\
\hline$d_{r t}(\mathrm{~mm})$ & 1.905 & 1.909 \\
\hline$d_{b}(\mathrm{~mm})$ & 0.2 & 0.2 \\
\hline
\end{tabular}

\subsection{Dimensional variants}

The original individual optimal design problem that needs to be solved is described formally in Eq. (7).

$$
\text { minimize } m
$$

$$
\begin{array}{rc}
\text { with respect to } & \mathbf{b}, \mathbf{h}, \mathbf{t} \\
\text { subject to } & 21 \mathrm{~Hz} \leq \omega_{1} \\
24 \mathrm{~Hz} & \leq \omega_{2} \\
d_{f t} & \leq 2.9 \mathrm{~mm} \\
d_{r t} & \leq 2.9 \mathrm{~mm} \\
d_{b} & \leq 0.2 \mathrm{~mm} \\
\sigma_{\max } & \leq 25 \mathrm{MPa}
\end{array}
$$

Here we consider a vehicle family of two variants, based on dimensional changes ( $c f$. Figure 4 ), and having the same objective functions and constraints.

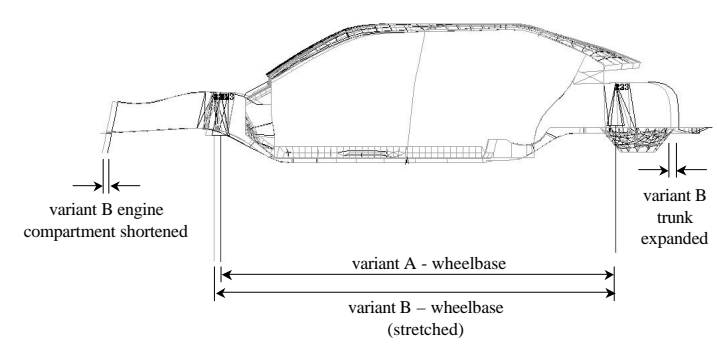

Figure 4: Vehicle body structure variants.

As shown in Figure 4, a second variant is generated by stretching the wheelbase and trunk of the baseline vehicle. The engine compartment is shortened, and therefore a smaller engine (and lumped mass representing the engine) is assumed. The models will be correspondingly referred to as the short and long wheelbase body models. The corresponding null platform optima are summarized in Table 1.

The SPV is computed according to Eq. (5). The platform is then determined by the value of the SPV as described in Section 4. Based on the values of the SPV, 59 variables are selected for sharing. The total platform — in which all variables are 
Table 2: Optima for 59-variable and total platforms.

\begin{tabular}{|c|c|c|c|c|}
\cline { 2 - 5 } \multicolumn{1}{c|}{} & \multicolumn{2}{c|}{59 var. platform } & \multicolumn{2}{c|}{ total platform } \\
\hline variant & short & long & short & long \\
\hline mass $(\mathrm{kg})$ & 715.17 & 703.54 & 725.65 & 703.37 \\
\hline$\omega_{1}(\mathrm{~Hz})$ & 21.00 & 22.06 & 21.00 & 22.24 \\
\hline$\omega_{2}(\mathrm{~Hz})$ & 24.82 & 27.00 & 25.83 & 27 \\
\hline$d_{f t}(\mathrm{~mm})$ & 2.158 & 2.170 & 2.082 & 2.171 \\
\hline$d_{r t}(\mathrm{~mm})$ & 1.905 & 1.909 & 1.837 & 1.911 \\
\hline$d_{b}(\mathrm{~mm})$ & 0.2 & 0.2 & 0.191 & 0.2 \\
\hline
\end{tabular}

shared - is also computed in order to compare the usefulness of the approach to the maximum sharing penalty. Both resulting family optima are given in Table 2.

Overall, the family based on the 59-variable platform is close to the null platform: The optimal masses of both short and long wheelbase variants are almost identical to the corresponding null platform designs. The total platform-based long wheelbase variant is still close to the corresponding null platform variant, compared to a $10.5 \mathrm{~kg}$ difference in mass in the short wheelbase variants. A large number of variables may be shared with negligible sharing penalty, considering that the sharing penalty of each variant is less than $1.5 \%$ compared to the corresponding null platform variants. This can be traced to the fact that the variants do not have competing design objective functions, and that their geometric configurations are very similar ( $c f$. Figure 4). The combination of these two factors results in relatively close individual optima and family optima. The next study was subsequently devised to test the proposed methodology on a problem that does not present these features.

\subsection{Performance Variants}

We now look at variants based on the same geometric model (the short wheelbase model) and defined by different design objectives and constraints. Two variants with competing objectives are designed, denoted "stiff" and "light weight", respectively. In the former the designer aims at maximizing the stiffness of the structure to improve ride quality, while in the latter the goal is to minimize weight to improve fuel economy.

The flexibility $\varphi$ is defined as a weighted sum of the displacements in the three load cases considered (front and rear torsion, and bending). The weights approximate the ratios of the expected displacements ( $c f$. null platform optima in Table 1) in each load case, hence flexibility is computed as
Table 3: Optimal design problems and associated null platform optima.

\begin{tabular}{|c|c|c|}
\hline variant & lightWeight & stiff \\
\hline obj. & min mass & min flexibility \\
\hline var. & Beams: $b, h$ and $t$; Shells $t 66$ variables \\
\hline constr. & $15 \mathrm{~Hz} \leq \omega_{1}$ & $21 \mathrm{~Hz} \leq \omega_{1}$ \\
\cline { 2 - 3 } & $17 \mathrm{~Hz} \leq \omega_{2}$ & $24 \mathrm{~Hz} \leq \omega_{2}$ \\
\cline { 2 - 3 } & $d_{f t}, d_{r t} \leq 2.9 \mathrm{~mm}$ & mass $\leq 822 \mathrm{~kg}$ \\
\cline { 2 - 3 } & $d_{b} \leq 0.5 \mathrm{~mm}$ & - \\
\cline { 2 - 3 } & \multicolumn{2}{|c|}{$\sigma_{\max } \leq 25 \mathrm{MPa}$} \\
\hline ppt. & mass $=691.87 \mathrm{~kg}$ & $\varphi=4.4049 \mathrm{~mm}$ \\
\hline
\end{tabular}

follows:

$$
\varphi=d_{f t}+d_{r t}+10 d_{b}
$$

Each variant thus defined is then individually optimized to obtain a null platform design. The individual optima are subsequently used to compute the SPV following Eq. (5). The individual optimization problems and their corresponding optima are summarized in Table 3.

\subsection{Sharing Penalty Vector Computation and Platform Selection}

Each design variable with index $i$ has a corresponding sharing penalty $\mathrm{SPV}_{i}$. The design variables are then arranged in order of increasing penalty. To aid visualization, we represent a graph of the penalty versus the number of design variables shared. This is shown in Figure 5.

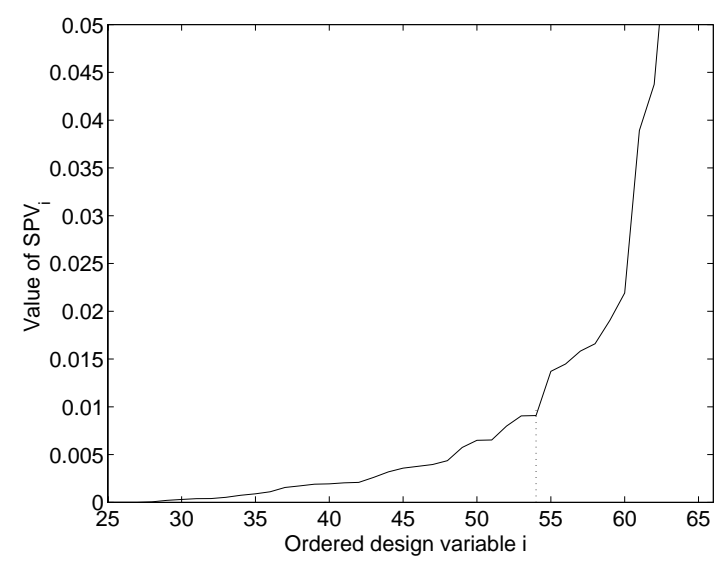

Figure 5: SPV vs. design variable number in order of increasing $\mathrm{SPV}_{i}$ value (performance variants).

We also compute the design of the family for the total platform. This represents an upper bound on the sharing penalty. The graph shows how the penalty remains low for the first 50 or so variables, then begins to increase sharply. We chose a 54- 
variable platform based on the fact that the curve presents an elbow at 54 variables ( $c f$. Figure 5).

Figure 6 is the representation of the whole Pareto set that generalizes the results in Table 4, which were obtained by minimizing the distance between the Pareto set and the null platform optimum (the point of coordinates $(1,1)$ ). The 54 variable platform shares all but $18 \%$ of the variables, and presents a penalty of $0.6 \%$ for the stiff variant and $1.16 \%$ for the light weight variant. In contrast, the total platform presents a $1.4 \%$ penalty for the stiff variant and an $18.8 \%$ penalty for the light weight variant.

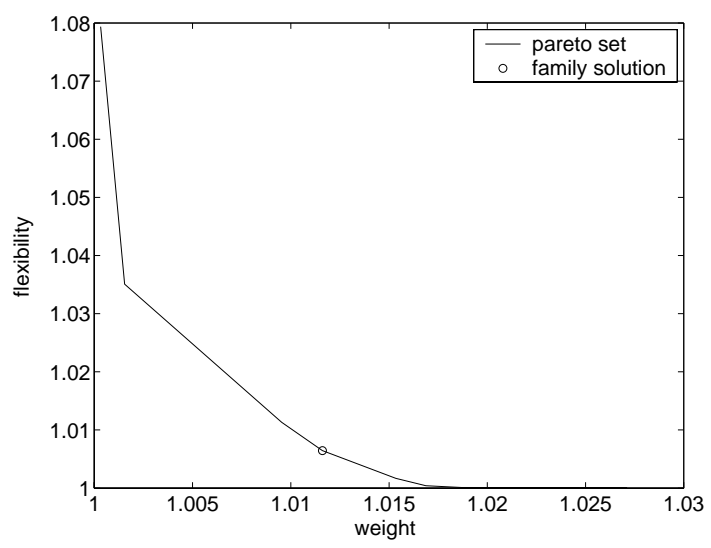

(a)

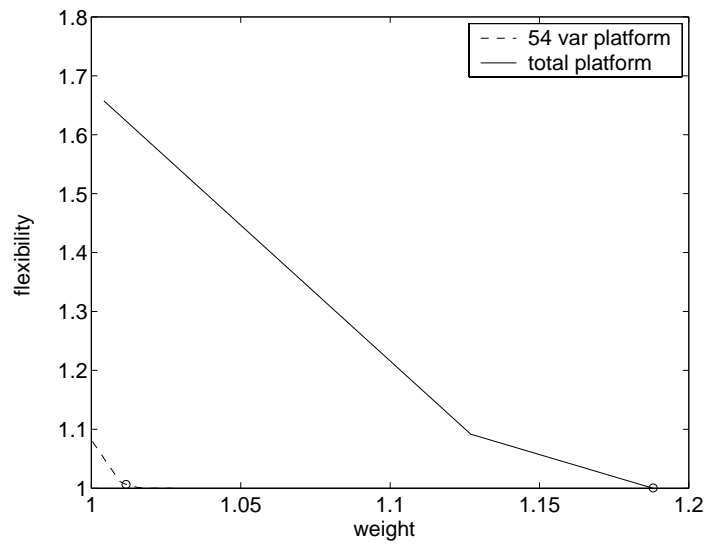

(b)

Figure 6: Pareto sets for the 54-variable and total platforms, expressed in relative sharing penalty. Figure 6a represents the Pareto set for the 54variable platform; Figure 6b contains both Pareto sets, the dashed curve corresponding to the Pareto set of the 54-variable platform. It appears smaller because of the difference in scale in the two graphs.

\subsection{Assumption Validation}

Some of the assumptions described in Section 3.1 can only be checked a posteriori. They were exam- ined using the 54-variable platform. The validity of each assumption for this platform is assessed below.

Assumptions 1 and 2 are automatically satisfied by the framework in which the case study was set up.

For Assumption 3, the distance between the platform designs is evaluated by computing the average of the relative difference in design variables $x_{i}^{s t, \circ}$ and $x_{i}^{l w, \circ}$ for all $i$, which is 0.187 . This gives us an idea of the relative difference between designs.

Assumption 5 is designed to avoid the case where a constraint that is inactive in the individual solution becomes active in the family solution, case which is not taken into account in the current derivation of Section 3.2. Here this assumption is violated in the sense that one constraint - out of 8 - that is active in the individual solution of the lightweight variant becomes inactive in the family solution. It is in agreement with the intuition that by adding equality constraints in the family design problem (the commonality constraints), it is likely that other constraints become inactive. However, this occurrence does not invalidate the conclusion of the derivation.

Assumption 4 however is not entirely satisfied. This assumption is very strong, therefore it was expected that issues would arise. The distance of the family solution to the convex hull of the individual solutions - the line between $\boldsymbol{x}^{s t, \circ}$ and $\boldsymbol{x}^{l w, \circ}$ - is 25.1 , the distance between these two points being 89.2. The projection of the family solution on the convex hull corresponds to an $\alpha$ of 0.23 (see Assumption 4), meaning that the family solution is closer to the "stiff" individual optimum. Further work must be accomplished to relax this assumption.

If the assumptions required by the approximation are satisfied, the SPV by itself provides an upper bound on the potential sharing penalty. Several platforms, chosen according to the order given by the SPV were computed, with a number of variables ranging from 30 to 65 . In Figure 7, the actual penalty for the objective functions of each variant - weight and flexibility — is plotted against the value of the upper bound given by the aggregate sum of the $\mathrm{SPV}_{i}$ that correspond to shared variables .

To further test the efficiency of the SPV approach, platforms were computed by sharing the variables in an order reversed to the one given by the SPV - a worst case scenario. In Figure 8 the actual penalty incurred in those platforms is plotted versus the penalty to the platforms de- 
Table 4: Comparison of null platform and 54-variable and total platform.

\begin{tabular}{|c|c|c|c|c|c|c|}
\hline Platform & \multicolumn{2}{|c|}{ null platform } & \multicolumn{2}{c|}{54 -variable platform } & \multicolumn{2}{c|}{ total platform } \\
\hline variant & stiff & l. weight & stiff & l. weight & stiff & l. weight \\
\hline mass $(\mathrm{kg})$ & 822 & 691.87 & 822 & 699.90 & 822 & 822 \\
$d_{f t}(\mathrm{~mm})$ & 1.581 & 2.429 & 1.595 & 2.270 & 1.607 & 1.607 \\
$d_{r t}(\mathrm{~mm})$ & 1.396 & 2.148 & 1.409 & 1.007 & 1.419 & 1.419 \\
$d_{b}(\mathrm{~mm})$ & 0.1427 & 0.2922 & 0.1429 & 0.2829 & 0.1443 & 0.1443 \\
flexibility $(\mathrm{mm})$ & 4.405 & 7.499 & 4.433 & 7.107 & 4.468 & 4.468 \\
\hline
\end{tabular}

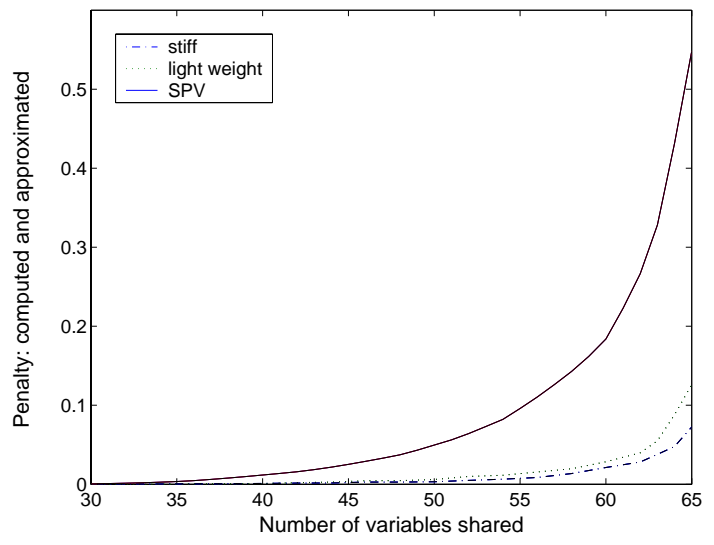

Figure 7: Actual sharing penalty vs. predicted by SPV (non dimensional).

signed according to the SPV. This figure demonstrates the gain in selecting a platform according to the process above. It also shows that if the assumptions are not entirely satisfied, the sharing penalty cannot be accurately predicted using the SPV. Had the assumptions been met, it would have been expected that the reverse SPV platform reached a plateau towards 65 variables.

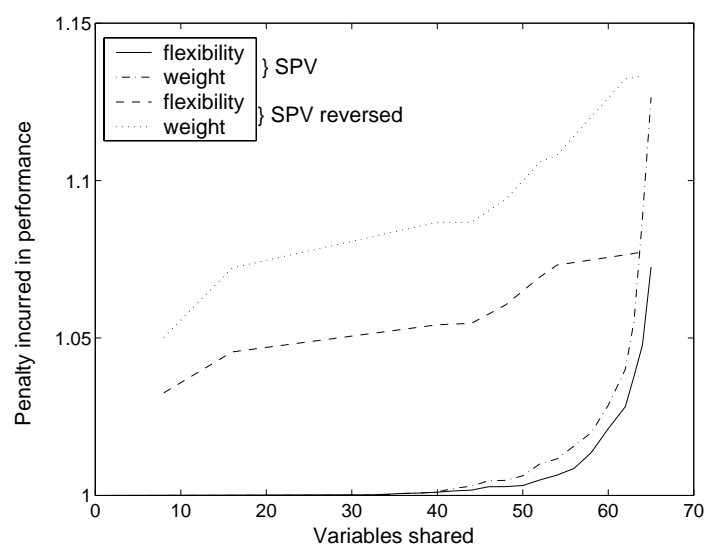

Figure 8: Actual penalty for both objective functions, for platforms computed according to the SPV and SPV reversed.

\section{Conclusions}

The methodology presented uses first-order information to compute a metric for measuring the penalty attributed to sharing specific components. This analysis can be used to identify which components should or should not be part of the product platform and becomes essential when the number of sharing combinations becomes too high to search exhaustively. Further examination of assumptions is a priority in future work. The methodology also needs to be tested on product families including more than two products. The desire is to enhance and validate the approach as a general methodology for reducing the platform design problem and for providing the designer with guidance for choosing the "optimal" platform.

\section{ACKNOWLEDGMENTS}

This research was partially supported by the $\mathrm{Au}$ tomotive Research Center, a US Army Center of Excellence in Modeling and Simulation of Ground Vehicles, by a Dual-Use Science and Technology Project and by the General Motors Collaborative Research Lab at the University of Michigan. This support is gratefully acknowledged. The authors also wish to thank Joe Donndelinger, Devadatta Kulkarni, and Robin Stevenson for their many helpful ideas and suggestions. The opinions expressed in this paper are solely those of the authors and do not reflect acknowledgement or acceptance by the sponsors.

\section{REFERENCES}

Meyer M.H., and Lehnerd A.P., 1997, The Power of Product Platforms, Free Press.

Ericsson A., and Erixon, G., 1999, Controlling Design Variants: Modular Product Platforms, ASME Press.

Simpson T.W., Maier J.R.A., and Mistree F., 1999, "A Product Platform Concept Exploration Method for Product Family Design," Proceedings of the 1999 ASME Design En- 
gineering Technical Conferences, Paper No. DTM-8761, Las Vegas, NV.

Conner C.G., De Kroon J.P., and Mistree F., 1999, "A Product Variety Tradeoff Evaluation Method for a Family of Cordless Drill Transmissions," Proceedings of the 1999 ASME Design Engineering Technical Conferences, paper DAC-8625, Las Vegas, NV.

Messac A., Martinez M.P., and Simpson T.W., 2000, "Effective Product Family Design Using Physical Programming and the Product Platform Concept Exploration Method," Proceedings of the 2000 ASME Design Engineering Technical Conferences, paper DAC-14252, Baltimore, MD.

Nayak R.U., Chen W., and Simpson T.W., 2000, "A Variation-Based Methodology for Product Family Design," Proceedings of the 2000 ASME Design Engineering Technical Conferences, paper DAC-14264, Baltimore, MD.

Gonzalez-Zugasti J.P., Otto K.N., and Baker J.D., 1998, "A Method for Architecting Product Platforms with an Application to Interplanetary Mission Design," Proceedings of the 1998 ASME Design Engineering Technical Conferences, paper DAC-5608, Atlanta, GA.

Gonzalez-Zugasti J.P. and Otto K.N., 2000, "Modular Platform-Based Product Family Design," Proceedings of the 2000 ASME Design Engineering Technical Conferences, paper DAC14238, Baltimore, MD.

Fujita K., and Yoshida H., 2001, "Product Variety Optimization: Simultaneous Optimization of Module Combination and Module Attributes," Proceedings of the 2001 ASME Design Engineering Technical Conferences, paper DAC-21058, Pittsburgh, PA.

Siddique Z., Rosen D.W, and Wang N., 1998, "On the Applicability of Product Variety Design Concepts to Automotive Platform Commonality," ASME Design Engineering Technical Conferences, Paper No. 98-DETC/DTM-5661, Atlanta, GA.

Fellini R., Papalambros P.Y., and Weber T., 2000, "Application of a Product Platform Design Process to Automotive Powertrains," Proceedings of the 8th AIAA/NASA/USAF/ISSMO Symposium on Multidisciplinary Analysis and $O p$ timization, Paper No. 4849, Long Beach, CA.
Nelson S.A., Parkinson M.B., and Papalambros P.Y., 2001, "Multicriteria Optimization in Product Platform Design," Journal of Mechanical Design, 123(2):199-204.

Kokkolaras M., Fellini R., Kim H.M., Michelena N.F., and Papalambros, P.Y., 2002, "Extension of the Target Cascading Formulation to the Design of Product Families," Journal of Structural and Multidisciplinary Optimization, to appear.

Fenyes P.A, 2000, "Multidisciplinary Design and Optimization of Automotive Structures - A Parametric Approach," Proceedings of the 8th AIAA/NASA/USAF/ISSMO Symposium on Multidisciplinary Analysis and Optimization, Paper No. 4706, Long Beach, CA. 